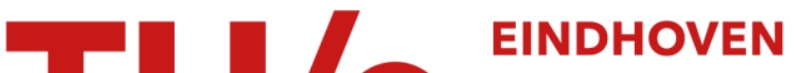

\section{Fractal dimension of tumor microvasculature by DCE-US: preliminary study in mice}

\section{Citation for published version (APA):}

Saidov, T., Heneweer, C., Kuenen, M. P. J., von Broich-Oppert, J., Wijkstra, H., de la Rosette, J. J. M. C. H., \& Mischi, M. (2016). Fractal dimension of tumor microvasculature by DCE-US: preliminary study in mice.

Ultrasound in Medicine and Biology, 42(12), 2852-2863. https://doi.org/10.1016/j.ultrasmedbio.2016.08.001

\section{Document license:}

TAVERNE

DOI:

10.1016/j.ultrasmedbio.2016.08.001

Document status and date:

Published: 01/12/2016

\section{Document Version:}

Publisher's PDF, also known as Version of Record (includes final page, issue and volume numbers)

\section{Please check the document version of this publication:}

- A submitted manuscript is the version of the article upon submission and before peer-review. There can be important differences between the submitted version and the official published version of record. People interested in the research are advised to contact the author for the final version of the publication, or visit the $\mathrm{DOI}$ to the publisher's website.

- The final author version and the galley proof are versions of the publication after peer review.

- The final published version features the final layout of the paper including the volume, issue and page numbers.

Link to publication

\section{General rights}

Copyright and moral rights for the publications made accessible in the public portal are retained by the authors and/or other copyright owners and it is a condition of accessing publications that users recognise and abide by the legal requirements associated with these rights.

- Users may download and print one copy of any publication from the public portal for the purpose of private study or research.

- You may not further distribute the material or use it for any profit-making activity or commercial gain

- You may freely distribute the URL identifying the publication in the public portal.

If the publication is distributed under the terms of Article 25fa of the Dutch Copyright Act, indicated by the "Taverne" license above, please follow below link for the End User Agreement:

www.tue.nl/taverne

Take down policy

If you believe that this document breaches copyright please contact us at:

openaccess@tue.nl

providing details and we will investigate your claim. 

Copyright (C) 2016 World Federation for Ultrasound in Medicine \& Biology Printed in the USA. All rights reserved $0301-5629 / \$$ - see front matter

\title{
FRACTAL DIMENSION OF TUMOR MICROVASCULATURE BY DCE-US: PRELIMINARY STUDY IN MICE
}

\author{
Tamerlan Saidov, $*$ Carola Heneweer, ${ }^{\dagger \dagger}$ MaArten Kuenen, ${ }^{*} §$ Julian von Broich-Oppert ${ }^{\dagger}$ \\ Hessel Wijkstra, ${ }^{\S}$ Jean de la Rosette, ${ }^{\S}$ and Massimo Mischi* \\ * Department of Electrical Engineering, Eindhoven University of Technology, Eindhoven, The Netherlands; ${ }^{\dagger}$ Clinic of \\ Radiology and Neuroradiology, University Hospital Schleswig-Holstein, Kiel, Germany; ${ }^{\ddagger}$ Institute for Diagnostic and \\ Interventional Radiology, University Hospital Cologne, Cologne, Germany; and ${ }^{\S}$ Department of Urology, The Academic \\ Medical Center, Amsterdam, The Netherlands
}

(Received 6 October 2015; revised 29 July 2016; in final form 1 August 2016)

\begin{abstract}
Neoangiogenesis, which results in the formation of an irregular network of microvessels, plays a fundamental role in the growth of several types of cancer. Characterization of microvascular architecture has therefore gained increasing attention for cancer diagnosis, treatment monitoring and evaluation of new drugs. However, this characterization requires immunohistologic analysis of the resected tumors. Currently, dynamic contrastenhanced ultrasound imaging (DCE-US) provides new options for minimally invasive investigation of the microvasculature by analysis of ultrasound contrast agent (UCA) transport kinetics. In this article, we propose a different method of analyzing UCA concentration that is based on the spatial distribution of blood flow. The well-known concept of Mandelbrot allows vascular networks to be interpreted as fractal objects related to the regional blood flow distribution and characterized by their fractal dimension (FD). To test this hypothesis, the fractal dimension of parametric maps reflecting blood flow, such as UCA wash-in rate and peak enhancement, was derived for areas representing different microvascular architectures. To this end, subcutaneous xenograft models of DU-145 and PC-3 prostate-cancer lines in mice, which show marked differences in microvessel density spatial distribution inside the tumor, were employed to test the ability of DCE-US FD analysis to differentiate between the two models. For validation purposes, the method was compared with immunohistologic results and UCA dispersion maps, which reflect the geometric properties of microvascular architecture. The results showed good agreement with the immunohistologic analysis, and the FD analysis of UCA wash-in rate and peak enhancement maps was able to differentiate between the two xenograft models $(p<0.05)$. (E-mail: t.a.saidov@ gmail.com) (c) 2016 World Federation for Ultrasound in Medicine \& Biology.
\end{abstract}

Key Words: Contrast-enhanced ultrasound, Fractals, Immunohistology, Angiogenesis, Cancer, Dispersion, Perfusion, Microvascular Architecture.

\section{INTRODUCTION}

Angiogenesis (and neovascularization) plays an important role in the growth and development of many types of solid tumors. It leads to the formation of a chaotic, dense network of irregular microvessels and is often a good predictor of cancer aggressiveness (Brawer 1996; Carmeliet and Jain 2000; Folkman et al. 1989). Angiogenesis is therefore a relevant imaging marker and promising prognostic indicator for cancer localization and diagnosis (Russo et al. 2012). Currently, the characterization of an angiogenic network requires an

Address correspondence to: Tamerlan Saidov, SPS Group, Department of Electrical Engineering, TU/e, De Zaale, 5600MB Eindhoven, The Netherlands. E-mail: t.a.saidov@gmail.com invasive procedure: analysis of microvascular density (MVD) from immunohistologic sections of resected tumors (Bigler et al. 1993; Heneweer et al. 2011; Russo et al. 2012). Non-invasive methods enabling characterization of angiogenic structures can therefore make an important contribution to cancer diagnostics, monitoring of treatment and evaluation of new (anti-angiogenic) drugs.

Currently, dynamic contrast-enhanced ultrasonography (DCE-US) is a widely employed imaging technique that makes use of ultrasound contrast agents (UCAs). UCAs are gas microbubbles encapsulated in a biocompatible shell that are injected intravenously and are easily detected by contrast-specific ultrasound imaging due to their high echogenicity and non-linear behavior (de Jong et al. 2000; Ferrara et al. 2000). Because they are 
only a few micrometers in diameter, UCA microbubbles can flow through the tiniest microvessels. Their use is therefore promising for non-invasive cancer detection by analysis of the microvasculature (Cosgrove 2003; Kuenen et al. 2011; Russo et al. 2012). In the literature, several approaches have been proposed for the assessment of cancer microvasculature by DCE-US. They are based on the analysis of features related to the change in UCA concentration over time, referred to as the time-intensity curve (TIC). All of these methods are based on a linear (or linearized) relationship between UCA concentration and measured acoustic intensity (Rognin et al. 2008).

The features of an estimated TIC relate to either blood perfusion or UCA dispersion kinetics, both of which are considered good markers for reflecting changes in the microvasculature due to the presence of cancerous angiogenic processes (Cosgrove 2003; Kuenen et al. 2011). For perfusion estimation, temporal features like wash-in rate or peak enhancement are extracted from the measured TIC; UCA replenishment following a destructive ultrasound pulse can also be analyzed and employed to measure perfusion (Hudson et al. 2009; Linden et al. 2007; Wijkstra et al. 2004). Contrast ultrasound dispersion imaging (CUDI) has recently been proposed as an alternative method for non-invasive cancer localization by assessing dispersion kinetics during DCE-US imaging (Kuenen et al. 2011; Mischi et al. 2012; Smeenge et al. 2011). In its most promising implementation, the similarity among neighboring TICs was estimated as an indirect measure of the dispersion coefficient based on the convection-dispersion equation (Kuenen et al. 2013; Mischi et al, 2012).

Several key properties of the microvascular architecture, such as microvascular density, vessel tortuosity and multi-path trajectories, influence not only the dispersion kinetics of a diluted agent (Taylor 1953) but also regional blood flow distribution (Qian and Bassingthwaighte 2000). Therefore, the regional blood flow distribution, defined by the geometry of the vascular network, is also affected by the presence of cancerous angiogenic processes (Jain 1999). The well-known concept of Mandelbrot suggests that fractal bifurcating networks mimic the vascular tree (Mandelbrot 1983), enabling the characterization of angiogenic networks in terms of fractal mathematics. In fact, the concept of Mandelbrot was applied to characterize regional blood flow distribution and cancer grading in immunohistologic samples (Baish and Jain 2000; Qian and Bassingthwaighte 2000; van Beek et al. 1989). By studying resected tissue samples, the vascular networks were represented by a specific parameter (i.e., the fractal dimension [FD]). It was shown that higher levels of vascular disorder lead to higher values of FD, whereas more structured vasculature is represented by a lower FD (Karshafian et al. 2003; Qian and Bassingthwaighte 2000).

For FD assessment of regional blood flow distribution, tissue samples are filled with a specific indicator (compound) that binds to the epithelial cells of blood vessels; the intensity of the bonded indicator is assumed to correlate with blood vessel size and density and, therefore, to flow (Qian and Bassingthwaighte 2000). Typically, FD assessment is based on images of indicator binding to the targeted cells. The image intensity is then assumed to correspond with the indicator density (Baish and Jain 2000; Qian and Bassingthwaighte 2000).

In this article, we evaluated the potential of FD to characterize microvascular networks by combining the aforementioned approaches with non-invasive DCE-US imaging. To this end, DCE-US was used to define suitable TIC parameters reflecting blood flow. In this study, peak intensity (PI) and wash-in rate (WiR) were measured. The method was tested with two subcutaneous (SC) xenograft mouse models of human prostate cancer (PCa) cell lines: DU-145 and PC-3. These models are characterized by a marked difference in MVD distribution: SC PC-3 develops a spatially homogenous vascular network, whereas SC DU-145 forms a core with higher MVD compared to the periphery (Heneweer et al. 2011; Saidov et al. 2012). For validation, the method was compared with immunohistologic MVD and FD assessment as well as with the recently proposed CUDI assessment based on the coherence between neighbor TICs measured by DCE-US (Kuenen et al. 2013; Mischi et al. 2012).

\section{MATERIALS AND METHODS}

\section{Data acquisition}

The animal experiments were performed at the University Hospital of Schleswig-Holstein in Kiel, Germany, in compliance with the Institutional Animal Care and Use Committee guidelines. The method for DCE-US-based FD estimation was developed and tested at the Eindhoven University of Technology in Eindhoven, The Netherlands.

Two types of PCa cell lines, DU-145 and PC-3, were implemented on SC xenograft models (Heneweer et al. 2011). Fourteen mice were injected subcutaneously with cancer cells (six with DU-145 and eight with PC3 ). Once the tumors had developed (14 d), all of the mice were administered a 0.1-mL bolus of MicroMarker Non-Targeted Contrast Agent Kit (VisualSonics Inc., Toronto, Ontario, Canada) using a tail vein catheter, and the TICs of the UCA passage through the tumors were measured by pixels. Necrosis was observed in one xenograft mouse model (DU-145). This mouse was therefore excluded from further analysis. 
DCE-US imaging was performed with a Vevo 2100 imaging system (VisualSonics Inc.) equipped with an MS-250 probe using contrast-specific imaging (power modulation) at $18 \mathrm{MHz}$ with axial resolution of about $0.08 \mathrm{~mm}$. The acquisition frame rate was $10 \mathrm{~Hz}$. A low mechanical index equal to 0.01 was adopted to prevent microbubble disruption. Separate in vitro measurements were performed to establish the relationship between UCA concentration and image intensity according to the procedure described by Mischi et al (2003) and Kuenen et al (2011). A logarithmic relationship between contrast agent concentration and image intensity was observed $\left(\mathrm{R}^{2}=0.98\right)$. This logarithmic relationship was compensated (linearized) to ensure that subsequent analysis was based on a linear measure of UCA concentration. An example of linearized TIC is presented in Figure 1.

After imaging, $50 \mu \mathrm{L}$ of tomato lectin fluorescein isothiocyanate (FITC; Vector Laboratories, Burlingame, CA, USA) were injected via a tail vein catheter in order to determine the MVD maps by immunohistology,. The animals were killed after 8 min of circulation. The tumors were then resected, embedded within Optimal Cutting Temperature compound (Tissue Tek, Sakura Finetek, Torrance, CA, USA) in cryomolds and stored at $-80^{\circ} \mathrm{C}$. Tissue sections $5 \mu \mathrm{m}$ thick were cut from the tumor and imaged with a fluorescence microscope (Axiovert, Zeiss, Oberkochen, Germany). The bound tomato lectin FITC normalized (between 0 and 1) grayscale intensity maps were taken as a measure of MVD (Heneweer et al. 2011). In seven mice, tomato lectin binding failed, and no fluorescence was observed. For these mice (two DU-145 and five PC-3), new tissue sections of 5- $\mu \mathrm{m}$ thickness were cut from the tumor and processed by CD31 staining. Slices were fixed $(200 \mathrm{~mL}$ of ice-cold acetone $+2 \mathrm{~mL}$ of a $30 \%$ solution of $\mathrm{H}_{2} \mathrm{O}_{2}$ ) for $10 \mathrm{~min}$.
After rinsing three times for 5 min with phosphatebuffered saline (PBS), non-specific binding was blocked with $4 \%$ bovine serum albumin in PBS for 20 min. Then the anti-CD31 antibody (BD Pharmingen 558736; BD Biosciences, San Jose, CA, USA) was added for $45 \mathrm{~min}$ in a $1 \%$ solution of bovine serum albumin in PBS using a dilution of 1:250. A non-specific IgG2a rat antibody was used in the same way as an isotype control. After rinsing three times for $5 \mathrm{~min}$ with PBS, the anti-ratAlexa 488 antibody (Molecular Probes A21210; Thermo Fisher Scientific Inc., MA, USA) was applied in the dark in a dilution of 1:1000 in PBS for 30 min. After rinsing three times with PBS, the slices were mounted with FluorSave (Merck Millipore Corporation, Darmstadt, Germany) and stored in the dark at $4{ }^{\circ} \mathrm{C}$. New fluorescence imaging was then performed using the same equipment. The MVD map was associated with the intensity (gray level) of the resulting digital image, which was lowpass filtered and normalized between 0 and 1 .

Regional blood flow and fractal dimension. The objective of this study is to investigate vascular heterogeneity by assessing the relative dispersion (RD) of the regional blood flow distribution. The RD is defined as the ratio between the standard deviation and mean of the regional blood flow distribution; RD is therefore a measure of spatial heterogeneity (Qian and Bassingthwaighte 2000).

For RD assessment by DCE-US, a parametric map that represents a measure of blood flow distribution must be generated. TIC parameters such as PI and WiR reflect properties of blood flow (Greis 2011). Therefore, these parameters are employed as flow measure to assess the RD of regional blood flow distribution. PI is defined as the TIC peak after preprocessing with a 5-s averaging filter. Appearance time (AT) is defined as the time when $10 \%$ of PI is first reached, and the time to peak (TP) is
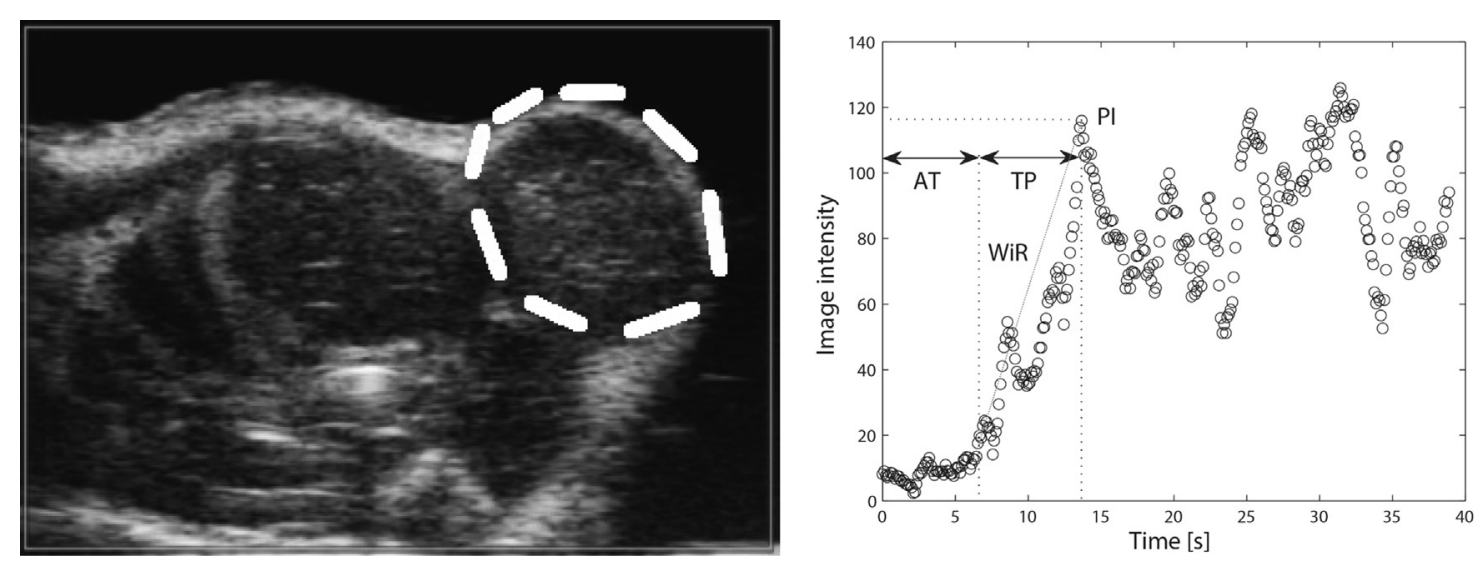

Fig. 1. Example of DCE-US imaging of a mouse impregnated with DU-145 cancer cells (left image; tumor is marked by white dashed line) and measured, linearized, filtered (5-s averaging) time-intensity curve (right). AT = appearance time; $\mathrm{PI}=$ peak intensity; TP = time-to-peak; WiR = wash-in-rate (PI/TP). 
the time between AT and PI. Finally, WiR is defined as PI/ TP, as shown in Figure 1. Figure 2 shows an example of PI and WiR parametric maps for an SC DU-145 and an SC PC-3 tumor; the corresponding normalized distributions (histograms) of the parameters are plotted in Figure.3. Normalization involves the intensity, which is scaled between 0 and 1 , as well as the distribution integral, which is equal to 1 .

Following the research of Bassingthwaighte et al. (Qian and Bassingthwaighte 2000; van Beek et al. 1989), FD is related to the relative dispersion of regional blood flow distribution, $\mathrm{RD}$. This relation is given in eqn (1):

$$
\frac{\mathrm{RD}(\mathrm{m})}{\mathrm{RD}\left(\mathrm{m}_{\mathrm{ref}}\right)}=\left(\frac{\mathrm{m}}{\mathrm{m}_{\mathrm{ref}}}\right)^{1-\mathrm{FD}}
$$

where FD is the fractal dimension (FD $>1$ ) and RD is the relative dispersion of the regional blood flow distribution for a given scale as determined by subsamples of size $\mathrm{m}$ (surface area). In practice, the RD can now be computed on the values representing the integral of the DCE-US parametric (flow) map over each tissue subsample. The ratio between the RD for tissue subsamples of size $m$ $(\mathrm{RD}[\mathrm{m}])$ and the $\mathrm{RD}$ for the reference subsamples of size $m_{\text {ref }}\left(R D\left[m_{r e f}\right]\right)$ is then calculated. The number of subsamples ( $\mathrm{n}$ ) into which a given tissue sample is divided is inversely related to $\mathrm{m}$, that is, $\mathrm{n} \propto 1 / \mathrm{m}$.
FD estimation. To estimate FD from eqn (1), a reference sample $m_{\text {ref }}$ and a method to define subsamples $m$ have to be defined. Generally, the choice of a reference sample is arbitrary; in this study, a sample was a zone covering the area to be investigated for its vascular heterogeneity. For consistency, we always chose $m_{\text {ref }}=1$ / 4, (i.e., a quarter of the entire zone) where 1 refers to the entire sample (zone); therefore, $\mathrm{m} \in[0,1]$. To optimize the computation time, the algorithm implemented in this study selected $m$ as represented in eqn (2):

$$
\mathrm{m}=\frac{1}{2^{2 \mathrm{k}}},
$$

where $\mathrm{k}=1,2,3, \ldots$ is a scale factor. Figure 4 shows a schematic of few subsequent subdivisions (scalings) for increasing $\mathrm{k}$.

For the assessment of FD by model fitting of the parametric maps, the derivative operator $\mathrm{d} / \mathrm{dm}$ is applied to eqn (1), resulting in eqn 3 :

$$
\begin{aligned}
\mathrm{d} \ln (\mathrm{RD}) & =(1-\mathrm{FD}) \mathrm{d} \ln (\mathrm{m}) \Rightarrow \Delta \ln (\mathrm{RD}) \\
& \approx(1-\mathrm{FD}) \Delta \ln (\mathrm{m}) .
\end{aligned}
$$

A linear regression is then used to estimate FD in eqn (3). An example of the fitting is presented in Figure 5. The method was tested by dedicated simulation using a map with known FD. For that purpose, a binary a

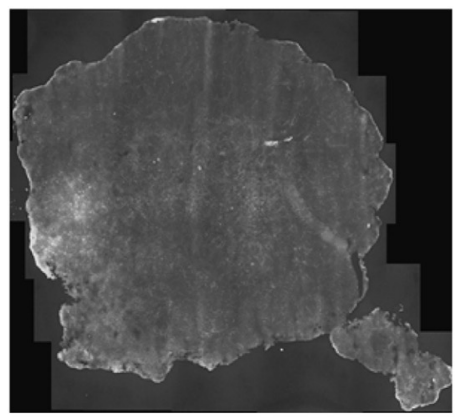

d

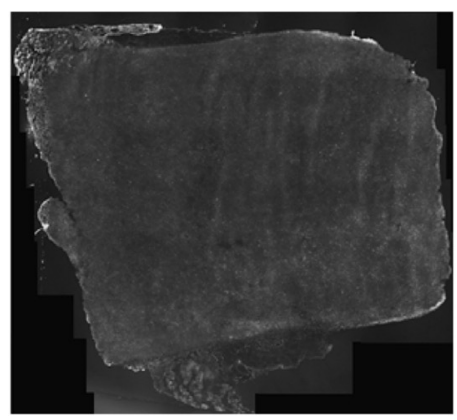

b

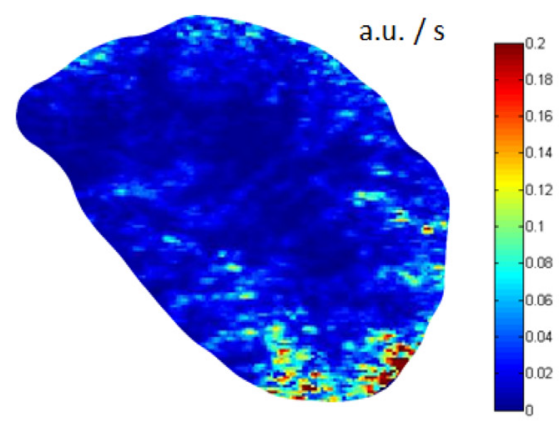

e

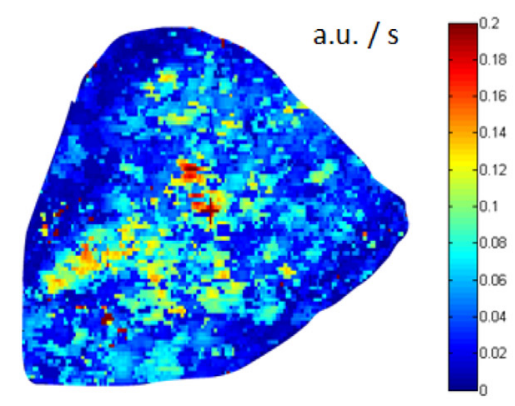

C

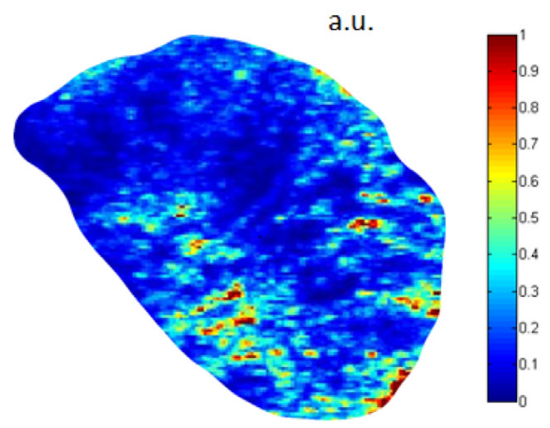

f

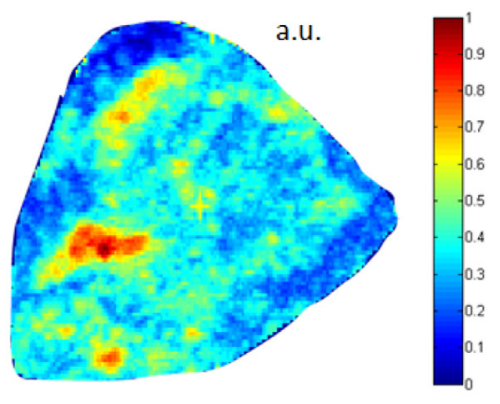

Fig. 2. Examples of tomato-lectin-FITC immunohistology of an SC DU-145 (a) and SC PC-3 (d) tumor, together with the corresponding DCE-US WiR (b and e) and PI (c and f) parametric maps, estimated for the whole tumor. a.u. = arbitrary units. 

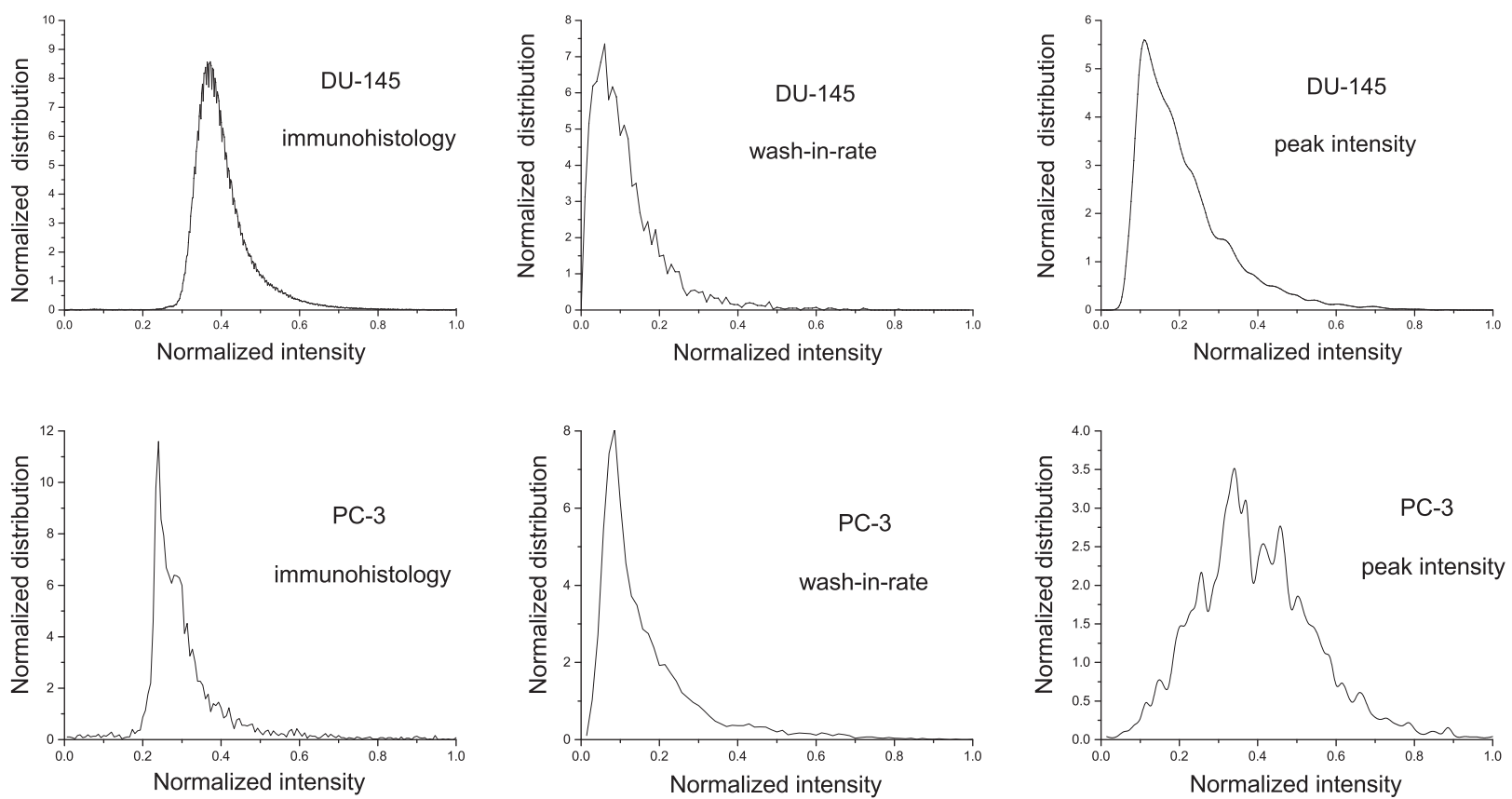

Fig. 3. Examples of normalized intensity distributions for immunohistology (left) and DCE-US parametric maps of WiR (center) and PI (right) corresponding to the parametric maps in Figure 2, calculated over the full surface of an SC DU-145 and PC-3 tumor.

(black and white) reference map with $\mathrm{FD}=1.5$ was used (from Fig. 1 in King et al. [1990]), and FD = 1.49 was obtained.

\section{Methodological considerations}

In real measurements, the observed $\mathrm{RD}\left(\mathrm{RD}_{\mathrm{obs}}\right)$ results from the RD of the vascular system (RD), the RD due to flow variation over time $\left(\mathrm{RD}_{\text {flow }}\right)$ and the $\mathrm{RD}$ due to measurement errors ( $\mathrm{RD}_{\text {error }}$ ) (Glenny and Robertson 1990). Assuming these contributions are independent, we can describe $R_{\text {obs }}$ as the sum shown in eqn (4) (Glenny and Robertson 1990):

$$
\mathrm{RD}_{\mathrm{obs}}^{2}=\mathrm{RD}^{2}+\mathrm{RD}_{\text {flow }}^{2}+\mathrm{RD}_{\text {error }}^{2}
$$

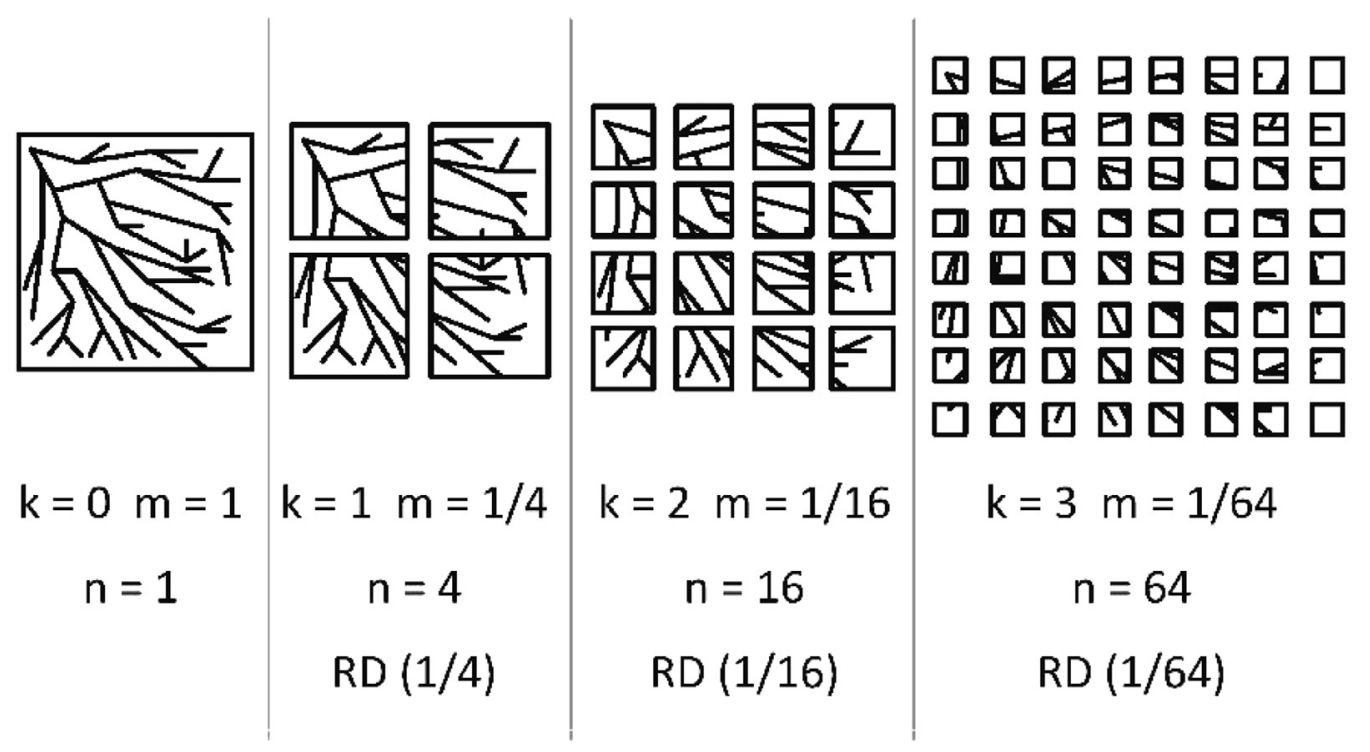

Fig. 4. Schematic representation of RD derivation for a given sample. From left to right, the scaling of the sample is depicted and $\mathrm{RD}$ is calculated as a function of $\mathrm{m} . \mathrm{k}=$ scale factor; $\mathrm{m}=$ surface area of subsample; $\mathrm{RD}=$ relative dispersion. 

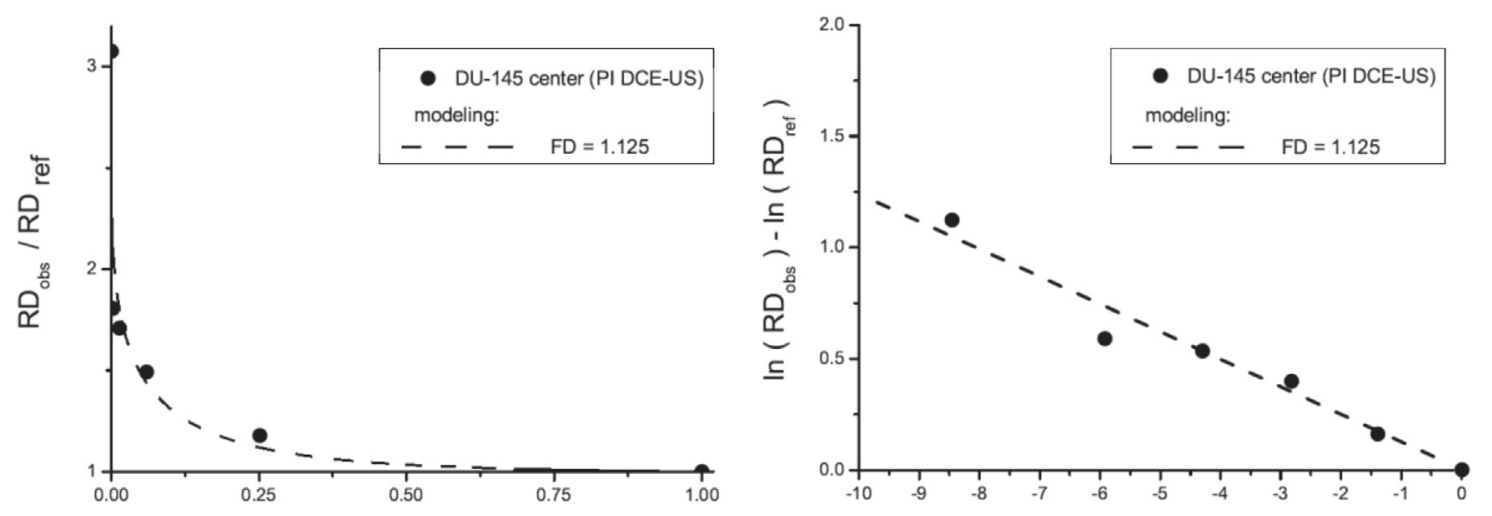

Fig. 5. Example of RD variation (PI by DCE-US in the central zone) as a function of sample size and FD assessment by curve fitting (dashed thick line) for a mouse impregnated with DU-145 cancer line. DCE-US = dynamic contrastenhanced ultrasonography; FD = fractal dimension; PI = peak intensity.

As we calculate RD over a single flow estimation with PI or WiR, which average flow variations, the RD introduced by flow variations is negligible and $\mathrm{RD}_{\text {flow }}$ $\simeq 0$. To guarantee that $\mathrm{RD}_{\text {obs }}$ reflects the actual relative distribution of regional blood flow $\mathrm{RD}$ (i.e., that it is minimally influenced by measurement errors), the following condition in eqn (5) must be satisfied:

$$
\mathrm{RD}_{\text {obs }}^{2} \approx \mathrm{RD}^{2} \gg \mathrm{RD}_{\text {error }}^{2} \text {. }
$$

The measurement errors relate to the possible effects of image resolution and noise on $\mathrm{RD}_{\mathrm{obs}}$. These errors can be significant when (i) the size of the tissue subsample (m) is close to that of a microvessel (unit of perfusion problem discussed in Glenny and Robertson [1990]), (ii) the tissue subsample is smaller than the image resolution and (iii) strong image noise is present. These three conditions are investigated below.

1. Mathematical (ideal) fractal objects keep fractal properties for any given scale, because zooming in does not influence the properties of the object. However, nonideal fractal objects carry fractal properties until a certain scale, which represents the smallest part of the object (maximal possible zooming) that can still be considered as representative of the entire object. In our context, this represents the unit of perfusion problem (Glenny and Robertson 1990). This problem occurs when the microvessel size is in the range of the tissue subsample size; that is, a given tissue subsample m contains only one (or a part of a) microvessel (unit of perfusion), but not a representative vascular network. In this case, the obtained FD does not represent the FD of a vascular network. In our images, the axial resolution of the scanner is $0.08 \mathrm{~mm}(80 \mu \mathrm{m})$ and the pixel size corresponds to $0.045 \mathrm{~mm}$, both of which are significantly larger than a capillary $(5-10 \mu \mathrm{m}$ in diameter).
2. To avoid the image resolution influencing the estimation of FD, a given tissue subsample of size m must always exceed the ultrasound scanner resolution. For simplicity and to avoid dealing with depth dependency, a homogeneous, isotropic resolution of $0.08 \mathrm{~mm}$ (axial resolution) was considered. In our DCE-US images, the pixel size is about $0.045 \times 0.045 \mathrm{~mm}^{2}$; that is, the ultrasound scanner resolution cell equals almost 4 pixels. Therefore, a tissue subsample must contain at least 4 pixels $(2 \times 2)$, i.e., $\mathrm{n}_{\max }=1 / \mathrm{m}\left(\mathrm{k}_{\max }\right)<\mathrm{N} / 4$, where $\mathrm{N}$ is the amount of pixels in the entire sample (when $m=1$ ) and $k_{\max }$ is the maximal value of the scale-factor $\mathrm{k}$. With respect to eqn (2), the condition for the scale-factor is shown in eqn (6):

$$
\mathrm{k}<\mathrm{k}_{\max }=\frac{1}{2}\left(\frac{\ln (\mathrm{N})}{\ln (2)}-2\right) .
$$

For the derived DCE-US parametric maps, the number of pixels in each map is approximately $20 \times 10^{3}$, and eqn (6) reads $k<6.14$. Therefore, to avoid resolution effects, we run k from 1 to 6 ; that is, eqn (3) is fitted through six points.

3. If conditions 1 and 2 are satisfied, the only error influencing $\mathrm{RD}_{\text {obs }}$ is noise, and eqn (5) can be rewritten in the form:

$$
\mathrm{RD}_{\text {obs }}^{2}=\mathrm{RD}^{2}+\mathrm{RD}_{\text {error }}^{2}=\mathrm{RD}^{2}+\mathrm{RD}_{\text {noise }}^{2} .
$$

DCE-US images are built on the US backscatter process. This process inherently results in speckle noise, which can be described statistically by a Rayleigh distribution (Thijssen and Mischi 2014) as shown in eqn (8), 
with $\alpha$ as a model parameter and $\mathrm{x}$ representing the variable (Papoulis and Pillai 1984):

$$
\mathrm{p}_{\mathrm{R}}=\frac{\mathrm{x}}{\alpha^{2}} \operatorname{Exp}\left[-\frac{\mathrm{x}^{2}}{2 \alpha^{2}}\right],
$$

Although noise in DCE-US shows more complex behavior, with a distribution that varies depending on the UCA concentration (Barrois et al. 2013; Kuenen et al. 2014), a Rayleigh approximation still holds for larger concentrations, such as at peak enhancement or toward the end of the wash-in phase (Bar-Zion et al. 2015). Therefore, assuming DCE-US gray level noise could be represented by Rayleigh noise, a dedicated simulation was implemented (see Appendix), proving that Rayleigh noise is reflected in parametric images of WiR and PI, whose estimation error is also well described by a Rayleigh distribution. As a result, eqn (7) can be represented by eqn (9), where $\mathrm{RD}_{\text {Rayleigh }}$ is the $\mathrm{RD}$ of a Rayleigh distribution:

$$
\mathrm{RD}_{\mathrm{obs}}^{2}=\mathrm{RD}^{2}+\mathrm{RD}_{\text {Rayleigh }}^{2},
$$

It can be shown that $\mathrm{RD}_{\text {Rayleigh }}$ is constant. In fact, the mean and standard deviation of the distribution in eqn (8) read, respectively:

$$
\mu_{\mathrm{R}}=\alpha \sqrt{\frac{\pi}{2}}, \sigma_{\mathrm{R}}=\alpha \sqrt{\frac{4-\pi}{2}} .
$$

The $\mathrm{RD}$ can be obtained as the standard deviation divided by the mean, i.e., $\mathrm{RD}_{\text {Rayleigh }}=\sqrt{4 / \pi-1}=$ const.
This proves the effect of Rayleigh noise on the estimated $\mathrm{RD}_{\text {obs }}$, referred to as $\mathrm{RD}_{\text {Rayleigh, }}$, is constant. As a result, eqn (5) can be rewritten in the form:

$$
\begin{aligned}
\left(\frac{\mathrm{RD}_{\text {obs }}(\mathrm{m})}{\mathrm{RD}\left(\mathrm{m}_{\mathrm{ref}}\right)}\right)^{2} & =\left(\frac{\mathrm{RD}_{\text {Rayleigh }}(\mathrm{m})}{\mathrm{RD}\left(\mathrm{m}_{\mathrm{ref}}\right)}\right)^{2}+\left(\frac{\mathrm{RD}(\mathrm{m})}{\mathrm{RD}\left(\mathrm{m}_{\mathrm{ref}}\right)}\right)^{2} \\
& =\text { const }+\left(\frac{\mathrm{RD}(\mathrm{m})}{\mathrm{RD}\left(\mathrm{m}_{\text {ref }}\right)}\right)^{2} .
\end{aligned}
$$

Applying a differential operator $\mathrm{d} / \mathrm{dm}$ to eqn (11) yields eqn (3). From this we conclude that DCE-US gray level noise makes only an additive constant contribution to the observed relative dispersion and does not affect the determination of FD.

\section{Validation}

To evaluate the potential of FD to characterize microvascular networks, the zones to be investigated (i.e., the zones to perform sequential subsampling) must be chosen. To this end, CUDI coherence analysis (Mischi et al. 2012) and immunohistology, aimed at the determination of the MVD maps, were performed; the results are presented in Figure 6.

CUDI coherence analysis was used as in Mischi et al. (2012) to assess the spatiotemporal similarity of each pixel TIC with the TICs measured in a surrounding ring-shaped kernel at distances between 0.25 and $1.0 \mathrm{~mm}$. The adopted similarity measure, referred to as coherence

\section{Immunohistology}
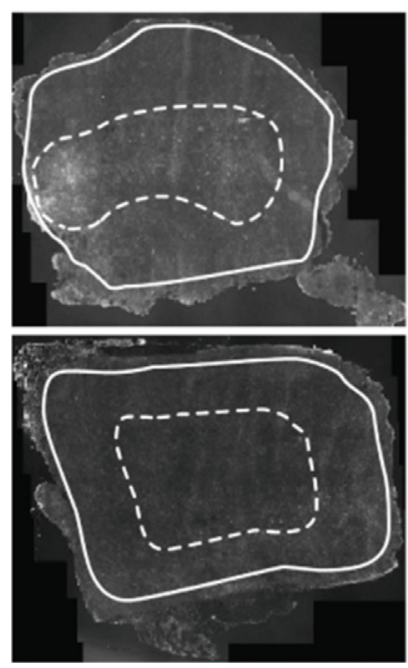

B-mode US
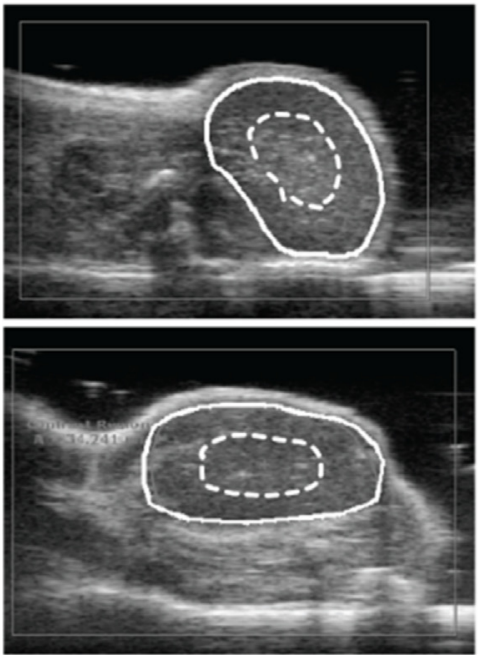

CUDI - Coherence

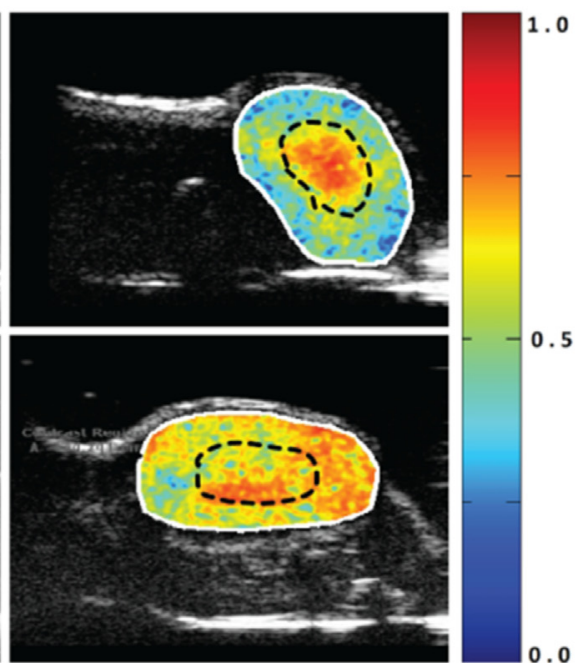

Fig. 6. Example of immunohistologic fluorescence images (left), B-mode (center) and contrast-specific (power modulation) mode with overlapped CUDI-coherence maps (right) of subcutaneous xenograft models for DU-145 (top) and PC-3 (bottom) human PCa lines. In comparison with PC-3, DU-145 xenografts show a heterogeneous coherence distribution, characterized by a high-coherence central region and a low-coherence peripheral region. CUDI $=$ contrast ultrasound dispersion imaging; $\mathrm{PCa}=$ prostate cancer. 
$(\rho)$, consists of the correlation coefficient of the TIC amplitude spectra in a fixed frequency range. This range, based on typical TIC frequency spectra, is taken between $0-0.5 \mathrm{~Hz}$. The coherence $\rho$ varies in a range of $[-1,1]$, that is, from anticorrelation $(r=-1)$, to uncorrelation $(r=0)$, up to maximum correlation $(r=1)$ of the power spectra. The adopted definition of coherence does not take into account TIC phase information and is therefore independent of mutual TIC delays. The levels of coherence were then used to generate CUDI-coherence maps (Fig. 6).

Based on the aforementioned methods, two zones were defined in each tumor: a central zone and a peripheral zone, as presented in Figure 6. According to the literature and to our MVD data (Heneweer et al. 2011; Saidov et al. 2012), the central zone in SC DU-145 tumors shows a higher vascularization with respect to the peripheral zone, while a higher degree of microvascular homogeneity is observed in SC PC-3 tumors. The central and the peripheral zone were defined in such a way that, in the case of DU-145, a threshold of $70 \%$ of the maximal value in CUDI-coherence (for the parameters PI and WiR) and MVD intensity (for immunohistology) levels were combined and used to distinguish between the zones. In the case of PC-3, the surface of the central zone is one third (corresponding to the average ratio between the two zones in SC DU-145 tumors) of the surface of the peripheral one with the same scaled-shape of the surface boundary.

For each tumor, the function $\mathrm{RD}_{\mathrm{obs}}(\mathrm{m})$ was obtained from both zones separately, with the scale factor $\mathrm{k}$ ranging up to 6 . The FD was estimated by curve-fitting eqn (3) to the obtained $\mathrm{RD}_{\text {obs }}(\mathrm{m})$ values, as shown in Figure 5. The linear regression method was applied for curve fitting. The FD was determined for the DCE-US flow maps (PI and WiR) as well as for the immunohistologic fluorescence maps. To investigate the difference in FD between the two predefined zones, the relative difference in FD between these zones was evaluated as:

$$
\frac{\Delta \mathrm{FD}}{\mathrm{FD}}=\frac{\mathrm{FD}_{\text {center }}-\mathrm{FD}_{\text {periphery }}}{\mathrm{FD}_{\text {center }}} .
$$

The same relative-difference approach was applied to the dispersion parameter derived from CUDI. For the purpose of validation, a relative difference as in eqn (12) was also estimated for WiR and PI maps. A twotailed Student's $t$-test was used to evaluate the statistical significance of the results $(p)$.

\section{RESULTS}

For DU-145 tumors, both immunohistology (MVD) and CUDI images show two zones with a lower (periphery) and a higher (center) intensity, while no significant difference between the two zones is found for PC-3 (Fig. 6). To compare the obtained FD results, relative differences $\Delta$ FD/FD are presented in Table 1 for immunohistology (MVD), WiR and PI maps. To estimate FD, the model in eqn (3) was fitted by linear regression; the overall coefficient of variation and correlation coefficient of the fits were 0.05 and $0.92(p<0.05)$, respectively.

In the chosen DCE-US parametric maps, PI-based FD shows the best capability to discriminate between central and peripheral zone for SC DU-145 and shows the highest similarity between these zones for SC PC-3. The difference in the microvasculature distribution across the tumor permits discrimination between SC DU-145 and SC PC-3 cancer lines $(p<0.05)$.

For validation purposes, Table 1 also reports the relative difference in PI and WiR between the two zones. In this case, the difference is not significant ( $p=0.61$ for $\mathrm{PI}$ and $p=0.14$ for $\mathrm{WiR}$ ).

\section{DISCUSSION}

Two DCE-US parameters reflecting the regional blood flow distribution, PI and WiR, were used to generate flow maps to be processed for the estimation of the FD. The presented results indicate good agreement of the estimated FD between the results obtained by immunohistology and CUDI, which shows an ability to distinguish between areas with different MVDs in agreement with the immunohistology. This is a promising result in the development of additional non-invasive tools for investigating tumor typing by means of FD.

The results, both from immunohistology as well as DCE-US, show that the SC DU-145 type of cancer develops two spatial regions with different FDs: a core with increased FD and an outer region with lower FD. SC PC-3 has no spatially different FD zones, indicating a more homogeneous vascularization. These results,

Table 1. Overall results (mean $\pm \mathrm{SD}$ ) of different imaging methods in DU-145 and PC-3 subcutaneous xenograft models in mice

\begin{tabular}{lcc}
\hline $\begin{array}{c}\text { Parameter (relative difference between the } \\
\text { central and peripheral zones) }\end{array}$ & DU-145 & PC-3 \\
\hline$\Delta$ FD/FD (immunohistology)* & $0.11 \pm 0.06$ & $0.01 \pm 0.02$ \\
$\Delta$ FD/FD (DCE-US, WiR-based)* & $0.18 \pm 0.11$ & $0.07 \pm 0.24$ \\
$\Delta$ FD/FD (DCE-US, PI-based)* & $0.17 \pm 0.13-0.04 \pm 0.11$ \\
$\Delta$ CUDI/CUDI & $0.37 \pm 0.08$ & $0.07 \pm 0.06$ \\
$\Delta$ MVD/MVD & $0.31 \pm 0.09$ & $0.10 \pm 0.12$ \\
$\Delta$ WiR/WiR & $0.29 \pm 0.25$ & $0.51 \pm 0.24$ \\
$\Delta$ PI/PI & $0.43 \pm 0.12$ & $0.45 \pm 0.11$ \\
\hline
\end{tabular}

CUDI $=$ contrast ultrasound dispersion imaging; DCE$\mathrm{US}=$ dynamic contrast-enhanced ultrasonography; $\mathrm{FD}=$ fractal dimension; MVD = microvascular density; PI = peak intensity; $\mathrm{WiR}=$ wash-in rate.

$* p<0.05$.

${ }^{\dagger} p<0.01$. 
Table 2. Immunohistologic results (mean $\pm \mathrm{SD}$ ) in DU-

145 and PC-3 subcutaneous xenograft models in mice comparing CD31 and tomato-lectin FITC staining

\begin{tabular}{|c|c|c|}
\hline $\begin{array}{l}\text { Staining method: Parameter } \\
\text { (relative difference between the central } \\
\text { and peripheral zones) }\end{array}$ & DU-145 & PC-3 \\
\hline $\begin{array}{l}\text { Tomato-lectin: } \Delta \text { FD/FD } \\
\text { (immunohistology)* }\end{array}$ & $0.11 \pm 0.05$ & $0.002 \pm 0.02$ \\
\hline CD31: $\Delta$ FD/FD (immunohistology)* & $0.11 \pm 0.06$ & $0.02 \pm 0.02$ \\
\hline Tomato lectin: $\Delta$ MVD/MVD ${ }^{\dagger}$ & $0.30 \pm 0.08$ & $0.09 \pm 0.13$ \\
\hline CD31: $\Delta$ MVD/MVD ${ }^{\dagger}$ & $0.32 \pm 0.09$ & $0.11 \pm 0.10$ \\
\hline
\end{tabular}

confirmed by immunohistologic analysis, agree with our previous analysis of SC DU-145 and PC-3 cancer lines by CUDI (Saidov et al. 2012), as well as with the previous literature (Heneweer et al. 2011).

The results show the ability of CUDI and DCE-US FD to discriminate between the different microvascular spatial distributions in the center and periphery of tumors. Direct analysis of the flow maps by PI and WiR could not produce the same results, confirming flow alone to be a poor indicator of neoangiogenic changes in the microvascular architecture.

In agreement with the concept of in dichotomously branching vascular networks (Qian and Bassingthwaighte 2000), a higher level of vascular disorder leads to higher values of FD, while more ordered vasculature shows lower FD. Although additional research is required to draw a definitive conclusion, the presented method shows a promising capability to assess the level of disorder of vascular networks. The obtained results also suggest an interesting correlation between vascular disorder assessed by FD, MVD assessed by immunohistology and UCA dispersion kinetics assessed by CUDI. In general, the ability to detect different degrees of vascularization by the presented methods may lead to new options for non-invasive tumor grading that support clinical decision making. To this end, an extensive clinical validation in humans is required.

Of particular interest are the improved FD results based on PI. The use of PI leads to better discrimination between the two cancer lines than the use of WiR. Other TIC parameters, such as mean transit time and TP have also been considered, but the resulting FD discrimination was lower than by WiR. Compared to the other parameters, PI is more related to vascular fraction than flow. We may therefore hypothesize that vascular fraction is a better parameter for characterizing underlying microvascular architectures based on the FD. However, to gain sufficient insight into these correlations, additional research is needed to investigate the relationship between the estimated FD and the main features characterizing microvascular architecture. To this end, not only MVD but also tortuosity and other forms of vascular irregularity should be investigated and correlated with the FD.

The results obtained by CUDI are superior by those obtained by FD. This might be because CUDI investigates kinetic processes rather than pure geometrical distributions. The methods are fundamentally different and can possibly underline different (complementary) features of microvascular architecture. Future research is therefore necessary for a deeper understanding of the relationship between these parameters and the main features of neoangiogenic microvascular networks, such as arteriovenous shunting, tortuosity and irregularity in size and bifurcations (Russo et al. 2012). Eventually, a combination of different parameters might provide more exhaustive information on underlying angiogenic processes. However, all these methods for characterization of microvascular architecture are limited by the need for a microvascular network and fail in the case of avascular, necrotic areas.
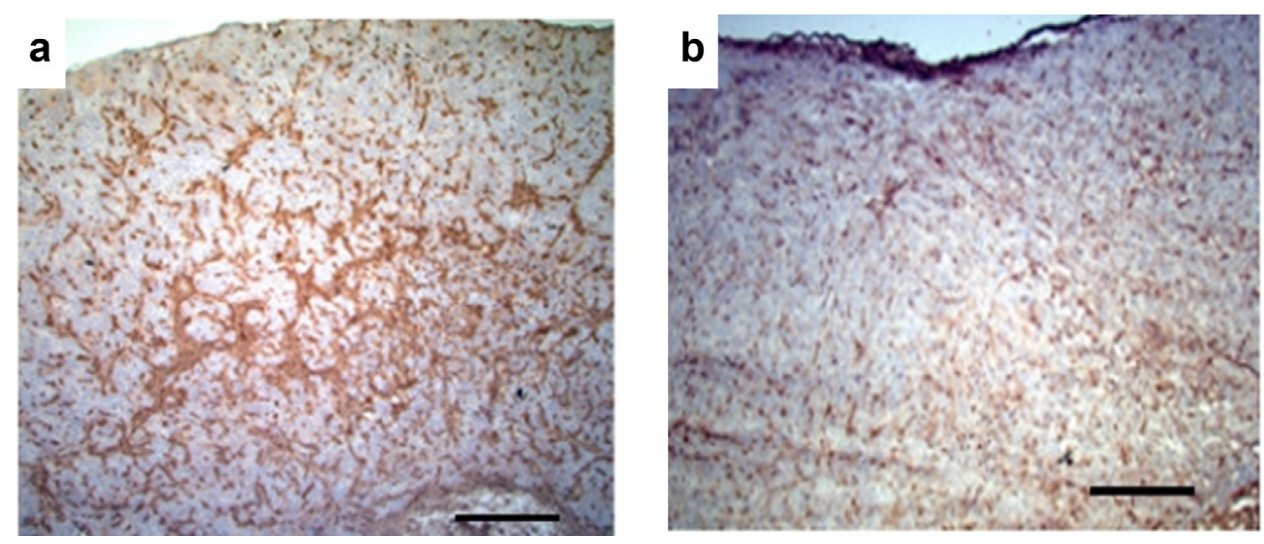

Fig. 7. Examples of CD31 staining in SC DU-145 (a) and PC-3 (b) tumors showing increased vascularization (red enhancement) in the core (central region) of DU-145. 

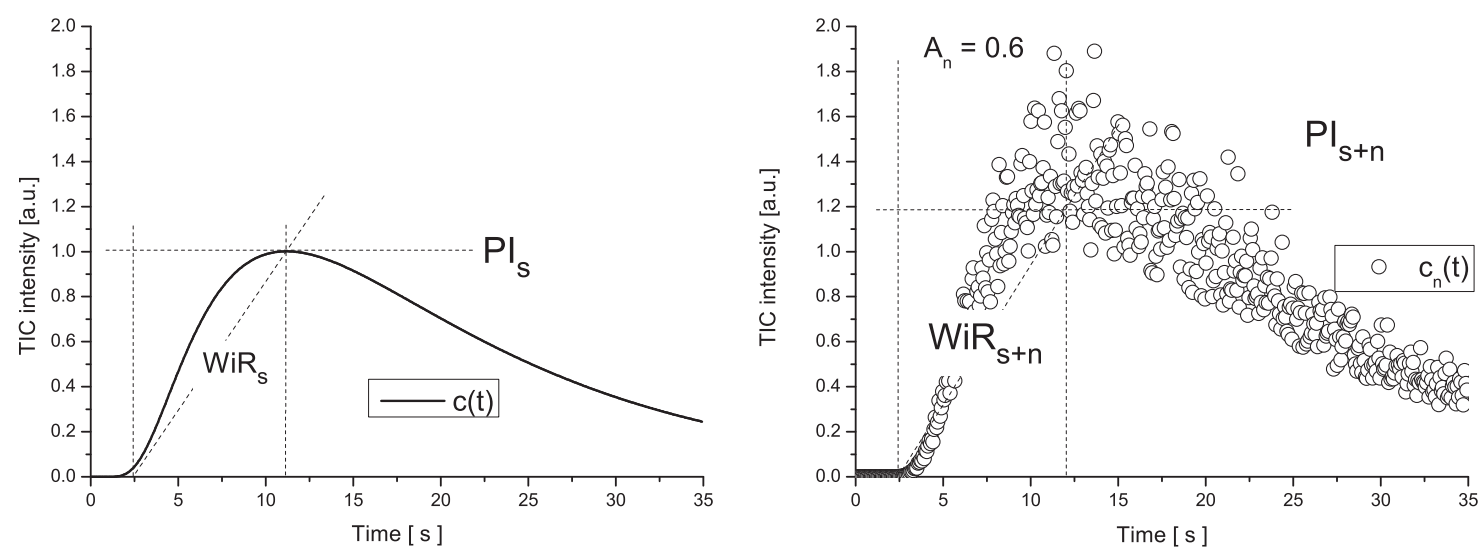

Fig. 8. An example of simulated TIC with (right) and without (left) noise. a.u. $=$ arbitrary units; $\mathrm{n}=$ noise; PI $=$ peak intensity; $\mathrm{s}=$ sound; TIC $=$ time-intensity curve; $\mathrm{WiR}=$ wash-in rate.

Validation was performed by comparison with immunohistology staining either by tomato lectin-FITC (seven mice) or by CD31 (seven mice). This was due to some difficulties in obtaining suitable fluorescence images by tomato lectin staining. Tomato lectin staining was preferred over CD31, as it enhances functional vessels only. To compare the two subgroups, the MVD and FD were also tested separately for the different immunohistologic tests. As shown in Table 2, the results are similar, and small differences may possibly be ascribed to the presence of closed-ended vessels, as highlighted by CD31 only. Figure 7 shows an example of CD31 staining for an SC DU-145 and an SC PC-3 tumor, confirming a hypervascular core for DU-145 in contrast to a homogeneous vascular distribution for PC-3.

Additional complexity in the validation procedure was posed by the determination of corresponding regions in the immunohistologic and DCE-US images. Although no automatic registration was employed, the scan was performed according to a standard protocol, with the probe aperture perpendicular to the mouse femur. This provided a reference point for manual alignment of the images. In the future, accurate registration can be achieved by using 3-D imaging, which would permit better image alignment even without employing automatic registration.

An additional advantage of 3-D over 2-D imaging also relates to the estimation of the RD. In a 2-D approach, all of the information across the elevation plane, which varies with image depth, is projected on one plane. This can affect the accuracy of the estimation. With a 3-D approach, the RD estimation can integrate higher-resolution information from the elevation plane, possibly gaining in accuracy and reliability of the estimates.

\section{CONCLUSIONS}

In this study, the theoretical requirements for correct fractal analysis have been discussed in relation to image characteristics and showed the acquisition settings were suitable for the proposed analysis. In future work, a thorough evaluation of the effects of the acquisition settings on the estimated FD should be conducted. The resolution
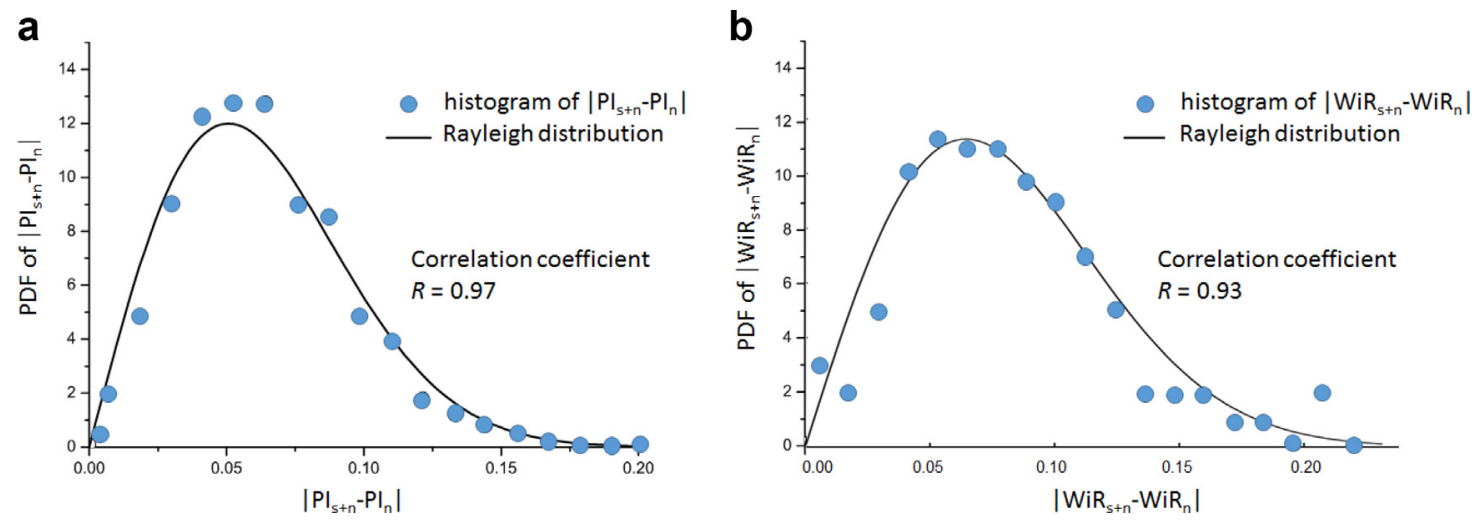

Fig. 9. Histograms of the TIC PI (a) and WiR (b) estimation error for noise amplitude $A_{n}=0.6$ with corresponding Rayleigh fitting. $\mathrm{n}=$ noise; $\mathrm{PDF}=$ probability distribution function; $\mathrm{PI}=$ peak intensity; $\mathrm{s}=$ sound; $\mathrm{WiR}=$ wash-in rate. 
anisotropy and depth dependency of ultrasound images should also be considered and evaluated in relation to the estimated FD. The results can be used to suggest optimal settings for performing fractal analysis. In particular, here the FD was estimated for entire predefined zones corresponding to different microvascular architectures. The application of the proposed method for diagnostic purposes would require the realization of an FD map from which variations in the underlying microvascular architecture could be detected. To this end, a kernel should be defined to span the entire parametric perfusion image and determine the FD at each position. A proper compromise should then be found between resolution in the resulting FD map, which improves with smaller kernel size, and FD estimation accuracy, which improves with larger kernel size (larger k).

\section{REFERENCES}

Baish JW, Jain RK. Fractals and cancer. Cancer Res 2000;60: 3683-3691.

Bar-Zion AD, Tremblay-Darveau C, Yin M, Adam D, Foster FS. Denoising of contrast-enhanced ultrasound cine sequences based on a multiplicative model. IEEE Trans Biomed Eng 2015;62: 1969-1980.

Barrois G, Coron A, Payen T, Dizeux A, Bridal L. A multiplicative model for improving microvascular flow estimation in dynamic contrast-enhanced ultrasound (DCE-US): Theory and experimental validation. IEEE Trans Ultrason Ferroelectr Freq Control 2013;60: 2284-2294.

Bigler SA, Deering RE, Brawer MK. Comparison of microscopic vascularity in benign and malignant prostate tissue. Hum Path 1993;24: 220-226.

Brawer MK. Quantitative microvessel density. A staging and prognostic marker for human carcinoma. Cancer 1996;78:345-349.

Carmeliet P, Jain RK. Angiogenesis in cancer and other diseases. Nature 2000;407:249-257.

Cosgrove D. Angiogenesis imaging-ultrasound. Br J Radiol 2003; 76(Spec No 1):S43-S49.

de Jong N, Frinking PJ, Bouakaz A, Ten Cate FJ. Detection procedures of ultrasound contrast agents. Ultrasonics 2000;38:87-92.

Ferrara KW, Merritt CR, Burns PN, Foster FS, Mattrey RF, Wickline SA. Evaluation of tumor angiogenesis with US: Imaging, Doppler, and contrast agents. Acad Radiol 2000;7:824-839.

Folkman J, Watson K, Ingber D, Hanahan D. Induction of angiogenesis during the transition from hyperplasia to neoplasia. Nature 1989; 339:58-61.

Glenny RW, Robertson HT. Fractal properties of pulmonary blood flow: Characterization of spatial heterogeneity. J Appl Physiol 1990;69: $532-545$.

Greis C. Quantitative evaluation of microvascular blood flow by contrast-enhanced ultrasound (CEUS). Clin Hemorheol Microcirc 2011;49:137-149.

Heneweer C, Holland JP, Divilov V, Carlin S, Lewis JS. Magnitude of enhanced permeability and retention effect in tumors with different phenotypes: ${ }^{89} \mathrm{Zr}$-Albumin as a model system. J Nucl Med 2011;52: 625-633.
Hudson JM, Karshafian R, Burns PN. Quantification of flow using ultrasound and microbubbles: A disruption replenishment model based on physical principles. Ultrasound Med Biol 2009;35:2007-2020.

Jain RK. Transport of molecules, particles, and cells in solid tumors. Annu Rev Biomed Eng 1999;1:241-263.

Karshafian R, Burns PN, Henkelman MR. Transit time kinetics in ordered and disordered vascular trees. Phys Med Biol 2003;48: 3225-3237.

King RB, Weissman LJ, Bassingthwaighte JB. Fractal descriptions for spatial statistics. Ann Biomed Eng 1990;18:111-121.

Kuenen MP, Herold IH, Korsten HH, de la Rosette JJ, Wijkstra H, Mischi M. Maximum-likelihood estimation for indicator dilution analysis. IEEE Trans Biomed Eng 2014;61:821-831.

Kuenen MP, Mischi M, Wijkstra H. Contrast-ultrasound diffusion imaging for localization of prostate cancer. IEEE Trans Med Imaging 2011;30:1493-1502.

Kuenen MP, Saidov TA, Wijkstra H, Mischi M. Contrast-ultrasound dispersion imaging for prostate cancer localization by improved spatiotemporal similarity analysis. Ultrasound Med Biol 2013;39: 1631-1641.

Linden RA, Trabulsi EJ, Forsberg F, Gittens PR, Gomella LG, Halpern EJ. Contrast enhanced ultrasound flash replenishment method for directed prostate biopsies. J Urol 2007;178:2354-2358.

Mandelbrot B. The fractal geometry of nature. San Francisco: WH Freeman and Co; 1983.

Mischi M, Kalker T, Korsten E. Videodensitometric methods for cardiac output measurements. EURASIP J Appl Signal Process 2003;5: 479-489.

Mischi M, Kuenen MP, Wijkstra H. Angiogenesis imaging by spatiotemporal analysis of ultrasound contrast agent dispersion kinetics. IEEE Trans Ultrason Ferroelectr Freq Control 2012;59:621-629.

Papoulis A, Pillai S. Probability, random variables, and stochastic processes. New York: McGraw-Hill; 1984.

Qian H, Bassingthwaighte J. A class of flow bifurcation models with lognormal distribution and fractal dispersion. J Theor Biol 2000; 205:261-268.

Rognin NG, Frinking P, Costa M, Arditi M. In-vivo perfusion quantification by contrast ultrasound: Validation of the use of linearized video data vs. raw RF data. In: Proceedings of the 2008 IEEE International Ultrasonics Symposium. Piscataway, NJ: IEEE; 2008. p. $1690-1693$.

Russo G, Mischi M, Sheepens W, de la Rosette JJ, Wijkstra H. Angiogenesis in prostate cancer: Onset, progression and imaging. BJU Int 2012;110:e794-e808.

Saidov T, Heneweer C, Kuenen M, Liesebach T, Wijkstra H, Mischi M. Contrast ultrasound dispersion imaging of different tumor types. In: Proceedings of the 2012 IEEE International Ultrasonics Symposium. Piscataway, NJ: IEEE; 2012. p. 2149-2152.

Smeenge M, Mischi M, Laguna Pes MP, de la Rosette JJ, Wijkstra H. Novel contrast-enhanced ultrasound imaging in prostate cancer. World J Urol 2011;29:581-587.

Taylor G. Dispersion of soluble matter in solvent flowing slowly through a tube. Proc R Soc Lond 1953;219:186-203.

Thijssen JM, Mischi M. Ultrasound imaging arrays. In: Panetta D, Marcello D, (eds). Comprehensive biomedical physics, vol. 2. Amsterdam: Elsevier; 2014. p. 323-341.

van Beek JH, Roger SA, Bassingthwaighte JB. Regional myocardial flow heterogeneity explained with fractal networks. Am J Physiol 1989;257:1670-1680.

Wagner RF, Smith SW, Sandrik JM, Lopez H. Statistics of speckle in ultrasound b-scans. IEEE Trans Sonics Ultrason 1983;30:156-163.

Wijkstra H, Wink MH, de la Rosette JJ. Contrast specific imaging in the detection and localization of prostate cancer. World J Urol 2004;22: 346-350. 


\section{APPENDIX}

\section{EFFECT OF SPECKLE ON THE PARAMETER ESTIMATION}

This appendix investigates the RD of the parameter (PI and WiR) estimation error.

First, we simulate a series of TICs $\left(20 \times 10^{3}\right.$ curves $)$ with the modified Local Density Random Walk model (mLDRW) as shown in eqn (A1) (Kuenen et al. 2011):

$$
\mathrm{c}(\mathrm{t})=\mathrm{A} \sqrt{\frac{\kappa}{2 \pi \mathrm{t}}} \operatorname{Exp}\left[-\frac{\kappa(\mathrm{t}-\mu)^{2}}{2 \mathrm{t}}\right],
$$

where $\mathrm{c}(\mathrm{t})$ is the intensity of a TIC as a function of time, A is the area under the curve, $\kappa$ is the dispersion parameter and $\mu$ is the mean transit time. This model was chosen because it provides a physical representation of the perfusion process based on the convective-diffusion equation (Kuenen et al. 2011). Within this simulation, we randomly vary (uniform distribution) the parameters $\kappa, \mu$ and $\mathrm{A}$ to mimic TICs measured in mice, that is $\kappa \in[0.05,0.15], \mu \in[10,25]$ and $\mathrm{A} \in$ $[15,30]$.
Second, multiplicative Rayleigh noise is applied to the simulated TICs (Wagner et al 1983):

$$
\mathrm{c}_{\mathrm{n}}(\mathrm{t})=\mathrm{A}_{\mathrm{n}} \mathrm{p}_{\mathrm{R}}(\alpha, \mathrm{x})
$$

with $c_{n}(t)$ being the resulting noisy TIC, $p_{R}$ the Rayleigh noise distribution and $A_{n}$ the noise amplitude relative to the intensity of the simulated TICs; that is, the values of $A_{n}$ are given as a fraction of the TIC maximal intensity. The value of $A_{n}$ is uniformly varied between 0.1 and 0.8 with 0.1 resolution, amply covering the range observed in the acquired data. For each value of $A_{n}, 100$ curves are generated. In eqn (2), $\alpha=\mathrm{c}(\mathrm{t}) 2 / \pi$ corresponds to the mean of the Rayleigh distribution and $\mathrm{p}_{\mathrm{R}}=\mathrm{c}(\mathrm{t})$ to the intensity of the TIC, while $\mathrm{x}$ is a uniformly distributed variable. An example of a simulated TIC is given in Figure 8.

For all simulated TICs, the parameters PI and WiR are estimated with and without noise, respectively; these are referred to as $\left(\mathrm{PI}_{\mathrm{s}+\mathrm{n}}\right.$, $\left.\mathrm{WiR}_{\mathrm{s}+\mathrm{n}}\right)$ and $\left(\mathrm{PI}_{\mathrm{s}}, \mathrm{WiR}_{\mathrm{s}}\right)$, respectively. The parameter estimation errors $\left|\mathrm{PI}_{\mathrm{s}+\mathrm{n}}-\mathrm{PI}_{\mathrm{s}}\right|$ and $\left|\mathrm{WiR}_{\mathrm{s}+\mathrm{n}}-\mathrm{WiR}_{\mathrm{s}+\mathrm{n}}\right|$ are then determined.

Finally, the normalized distribution (histogram) of the RD of the parameter estimation error is calculated and fitted with the Rayleigh distribution in eqn (8). An example is shown in Figure 9. For the investigated noise amplitude $\mathrm{A}_{\mathrm{n}}<0.8$, the correlation coefficient of the Rayleigh fit was $\mathrm{R}<0.86$, both for PI and WiR. Therefore, the estimation error is well approximated by a Rayleigh distribution. 